\title{
BMJ Open Developing an Australian multi-module clinical quality registry for gynaecological cancers: a protocol paper
}

\author{
Natalie Heriot (D) , ${ }^{1}$ Alison Brand, ${ }^{2,3}$ Paul Cohen, ${ }^{4,5,6}$ Sue Hegarty, ${ }^{7}$ Simon Hyde, ${ }^{8}$ \\ Yee Leung, ${ }^{4}$ John R Zalcberg, ${ }^{1,9}$ Robert Rome ${ }^{10}$
}

To cite: Heriot N, Brand A, Cohen $\mathrm{P}$, et al. Developing an Australian multi-module clinical quality registry for gynaecological cancers: a protocol paper. BMJ Open 2020;10:e034579. doi:10.1136/ bmjopen-2019-034579

- Prepublication history for this paper is available online. To view these files, please visit the journal online (http://dx.doi org/10.1136/bmjopen-2019034579).

Received 26 September 2019 Revised 16 January 2020 Accepted 07 February 2020

Check for updates

(c) Author(s) (or their employer(s)) 2020. Re-use permitted under CC BY-NC. No commercial re-use. See rights and permissions. Published by BMJ.

For numbered affiliations see end of article.

Correspondence to

Ms Natalie Heriot;

natalie.heriot@monash.edu

\section{ABSTRACT}

Introduction Gynaecological cancers collectively account for almost $10 \%$ of cancer diagnoses made in Australian women. The extent of variation in gynaecological cancer survival rates and treatment outcomes across Australia is not well documented. The purpose of the clinical quality registry described in this paper is to systematically monitor and improve quality of care provided to these women, and facilitate clinical process improvements to ensure better patient outcomes and greater adherence to best practice care. The registry infrastructure has been developed in conjunction alongside the inaugural ovarian, tubal and peritoneal (OTP) module, allowing for concurrent piloting of the methodology and one module. Additional tumour modules will be developed in time to cover the other gynaecological tumour types.

Method and analysis The National Gynae-Oncology Registry (NGOR) aims to capture clinical data on all newly diagnosed cancers of the uterus, ovary, fallopian tubes, peritoneum, cervix, vulva and vagina in Australia with a view to using these data to support improved clinical care and increased adherence to 'best practice'. Data are sourced from existing clinical databases maintained by clinicians and/or hospital gynaecological cancer units. A pilot phase incorporating only OTP cancers has recently been conducted to assess the feasibility of the registry methodology and assess the support of a quality initiative of this nature among clinicians and other key stakeholders. Ethics and dissemination The NGOR has received National Mutual Acceptance (NMA) ethics approval from Monash Health Human Research Ethics Committee (HREC), NMA HREC Reference Number: HREC/17/MonH/198. We also have approval from Mercy Health HREC and University of Tasmania HREC. Data will be routinely reported back to participating sites illustrating their performance against measures of agreed best practice. It is through this feedback system that the registry will support changes to quality of care and improved patient outcomes.

\section{INTRODUCTION}

The National Gynae-Oncology Registry (NGOR) is a new multi-modular clinical quality registry $(\mathrm{CQR})$ which aims to record diagnostic, treatment and outcome data of patients with newly diagnosed gynaecological cancers, and to use this information to monitor and minimise variation in care,

\section{Strengths and limitations of this study}

- We describe the purpose and process of developing a national clinical quality registry (CQR) for gynaecological cancers in Australia, using the ovarian, tubal and peritoneal cancer module as an example.

- The National Gynae-Oncology Registry was developed in line with the Australian Operating Principles for CQRs (developed in line by the Australian Committee of Safety and Quality in Health Care), and is managed within the Monash University School of Public Health and Preventive Medicine alongside approximately 30 other clinical registries.

- This is a clinician-driven initiative, using a model for data collection that should reduce long-term costs associated with registry operations through the use of previously collected clinical data.

- Gynaecological cancers are primarily managed by gynaecologic oncologists, who are the key clinical group behind this CQR. However, medical oncologists and other clinical specialities are vital to the management of these diseases. Their increased engagement and clinical expertise are vital to the registry's future.

improve patient outcomes and identify trends and potential gaps in service provision. The NGOR was developed by a group of likeminded clinicians in collaboration with the Cancer Research Program in Monash University's School of Public Health and Preventive Medicine in 2017, and has recently been piloted in Victoria, Tasmania and New South Wales. It was designed to assess outcomes and quality of care provided to women with ovarian and related cancers-the first module of the NGOR. Other modules of the registry will be developed over time, dependent on funding, and will be designed around anatomical tumour location and similarity in management and expected outcomes. The NGOR is currently in a transitional phase, working to develop these additional modules and adjusting the ovarian, tubal and peritoneal (OTP) module. The key reason for 
piloting one, rather than all modules, was to allow the registry infrastructure to be developed and piloted on a smaller scale. Once the registry has matured, additional modules can be piloted using-what will then be-an established infrastructure.

The registry will in time provide information about population-based patterns of care, identify variation in treatment patterns and outcomes across Australia, highlight factors that predict optimal treatment and outcome and prompt improved compliance with, best practicebased guidelines for the treatment of gynaecological cancers. The registry is also expected to improve our current understanding of these cancers by monitoring trends, providing infrastructure for future research studies and determining the clinical effectiveness of treatments in a 'real-world' setting.

\section{BACKGROUND}

\section{Gynaecological cancers in Australia}

Malignant tumours of the uterus, ovary, cervix, vulva and vagina collectively account for almost $10 \%$ of female cancer diagnoses made in Australia. ${ }^{7}$ In 2019, it was estimated that there will be 6454 new cases and 2040 deaths attributable to gynaecological cancers. ${ }^{1}$ There appears to be wide variation in the incidence of gynaecological cancers across Australia, with women of the lowest socioeconomic status (SES) being diagnosed at a more frequent rate compared with women of higher SES. ${ }^{2}$ Despite limited data, it has been estimated that Aboriginal and Torres Strait Islander women are 1.7 times more likely to be diagnosed with a gynaecological cancer when compared with non-Indigenous women, and are on average 10 years younger than their non-Indigenous counterparts (52 vs 62 years of age) when first diagnosed. ${ }^{2}$ The extent of variation in gynaecological cancer survival rates and treatment outcomes in hospitals across metropolitan and rural areas, and between states and territories is not well documented, although it has been reported anecdotally. Some variation is believed to be a consequence of differences in the prevalence of known risk factors, population characteristics, patient choice, availability and utilisation of diagnostic services. ${ }^{2}$ For example, $12 \%$ of women with epithelial ovarian cancer from an Australiawide population-based study (2002-2005) had a diagnostic delay of longer than 6 months. This was more likely for women residing in remote Australia and those with lower incomes. ${ }^{3}$ It is also suspected that some variation might be explained by differences in quality of care, and treatment availability across health services. A populationbased study of 1192 women diagnosed with ovarian cancer in 2005 showed that compared with women from relatively socioeconomically advantaged areas, women from relatively disadvantaged areas had a 21\% higher risk of dying during follow-up. A similar difference was seen for women living in regional-remote areas versus major cities. ${ }^{4}$ Recent, robust, real-world data to support or disprove reports of varied practice across Australia are currently not available, with the most recent patterns-ofcare in ovarian cancer study published in 2002, assessing care only within the State of Victoria between 1993 and $1995 .^{5}$

\section{Clinical quality registries}

CQRs are defined by the Australian Commission of Safety and Quality in Healthcare as datasets that systematically monitor the effectiveness and appropriateness of healthcare within specified clinical domains (ie, for a particular disease, condition or device). ${ }^{6}$ This is accomplished through the routine collection, analysis and reporting of health-related information from patient's medical records and other administrative data sources. Data are subsequently used to identify variation in performance in regards to predetermined quality indicators (QIs) that measure compliance against best practice, set benchmarks and inform improvements in healthcare. ${ }^{6}$ CQRs are not intended to completely capture all aspects of care, but rather form a solid foundation of data collected from the whole population of interest (or as close to $100 \%$ capture as possible). Thus, ideally a minimal dataset is collected, but these data have a definitive purpose, informing predetermined measures of adherence to 'best practice', that is, QIs. A CQR for gynaecological malignancies in Australia would be well placed to identify any variation in the management of these cancer types, and drive improvements in patient outcomes. Plans for such a registry have long been supported by the Australian Society of Gynaecologic Oncologists (ASGO).

\section{The inaugural module: OTP cancers}

Ovarian cancer is the 10th most common cancer in Australian women with an estimated 1510 new cases in 2019, accounting for a quarter of all gynaecological cancer diagnoses. ${ }^{7}$ It is also the most lethal gynaecologic malignancy with 1046 Australian women expected to die of the disease in $2019 .{ }^{7}$ Unlike many solid cancers for which the 5-year survival rate has collectively increased by $20 \%,{ }^{8}$ the 5 -year survival rate of women with epithelial ovarian cancer has changed little in recent decades with affected women having a $45.7 \%$ chance of surviving 5 years postdiagnosis (2011-2015 data). ${ }^{7}$ As there are no available effective screening tests for this disease, ${ }^{2}$ the symptoms are largely non-specific ${ }^{8}$ and patients remain asymptomatic until the disease is advanced ${ }^{9}$; it is difficult to diagnose early when the disease is confined to the ovary. ${ }^{2}$ Though the exact number of cases diagnosed at each stage is unknown due to the limited available data representing stage at diagnosis in Australia, a large proportion of cases $(>60 \%)^{410}$ are reportedly diagnosed at an advanced stage when there is multi-focal metastatic disease throughout the peritoneal cavity. ${ }^{2}$

Given that the most significant prognostic factor for ovarian cancer is stage at diagnosis, ${ }^{9} 11$ with early-stage disease considered to be 'curable' in $90 \%$ of cases, ${ }^{8}$ the research areas of greatest importance for improving survival are arguably a means to early detection as well as 
more timely treatment. ${ }^{8}$ Until such advances in preventive medicine are made, the greatest chance of improving survival for women diagnosed with ovarian cancer is to ensure they receive evidence-based high-quality care. Hence, the importance for this tumour type is to make up the first of the NGOR modules, and the focus of the pilot phase. Epithelial fallopian tube and peritoneal cancers were also included in the ovarian cancer module because of their clinical similarities in treatment and outcomes to epithelial ovarian cancer and because many epithelial 'ovarian' cancers originate in the distal fallopian tube. ${ }^{12}$

\section{Key causes of variation in patient outcomes}

\section{Patient and disease-related factors}

An Australian study published in 2014 investigated survival outcomes in Australian women diagnosed with epithelial ovarian cancer and found that poorer survival was strongly associated with increasing age and disease stage. ${ }^{4}$ It also found strong independent associations between poorer patient outcomes and the presence of ascites at diagnosis, multiple or severe comorbidities and regionalremote and relatively disadvantaged areas of residence. ${ }^{4}$ The authors' suggested socioeconomic and geographical differences in survival may be attributable in part to diagnostic delay and poorer access to treatment. ${ }^{4}$ The study also suggested major variations in unadjusted survival rates between states and territories. ${ }^{4}$ Although variations in survival rates did not achieve statistical significance, the study was likely underpowered to detect such differences.

BRCA1/2 mutations have been associated with overall survival, likely as a result of the high response rate to platinum-based chemotherapy; however, the exact nature of this effect is yet to be determined. ${ }^{13}$

\section{Treatment and disease management-related factors}

Several studies have demonstrated that better patient outcomes including survival are associated with complex surgical procedures being performed in specialised hospitals with higher case volumes. ${ }^{14}$ One study found that a hospital volume of $\geq 21$ cases of advanced ovarian cancer per year was significantly predictive of improved survival. ${ }^{15}$ Prolonged survival has frequently been observed when surgery is performed by gynaecological oncologists with specialised surgical training. ${ }^{8}{ }^{16-19}$ This is likely due to achieving optimal or 'complete' debulking (cytoreduction to no macroscopic residual disease) ${ }^{8}{ }^{8161719}$ Some authors have reported that gynaecologic oncologists are two times more likely to achieve this complete cytoreduction when compared with other surgeons. ${ }^{19}$ The extent of residual disease is an important prognostic factor with significant impact on overall survival, and has been recognised as such since $1975 .{ }^{16}{ }^{17}$ A meta-analysis by Bristow et al showed a survival benefit of $5.5 \%$ with every $10 \%$ increase in maximal cytoreduction. ${ }^{17}$

In addition to the goal of optimal debulking, appropriate staging of apparent early stage disease is vital as it will help determine whether adjuvant therapy is recommended. One trial found that inadequate staging occurred in about $66 \%$ of patients, with sampling of lymph nodes and diaphragmatic biopsy commonly omitted due to a lack of expertise and low case volume of the surgeon. ${ }^{14}$

\section{METHODS}

\section{Project history}

A national CQR for gynaecological cancers was first proposed in 2014; however, initial attempts to source seed funding for this registry were unsuccessful. As the reported 5-year survival for women diagnosed with gynaecological cancers remains stagnant, the need for realworld data on the suspected inconsistencies in quality of care became evident. Therefore, a multi-module registry was proposed using a novel and more cost-effective methodology to be piloted in gynaecological cancer specialist centres across Victoria, Tasmania and New South Wales. The aim was to try to align and then collate data from existing individual unit databases and pre-existing databases maintained by consultant gynaecologic oncologists.

\section{Governance structure}

The NGOR is a clinician-driven registry, with a focus on initial management and first-line treatment. A Steering Committee oversees the registry's development. Fifteen gynaecological oncologists and two medical oncologists sit on this committee alongside Monash University representatives and representatives from registry partners, providing clinical expertise and registry oversight. Funding for the pilot phase of the NGOR has been supported by Ovarian Cancer Australia, the ASGO and the CASS Foundation.

\section{Patient and public involvement}

Representatives from Ovarian Cancer Australia, the leading national organisation that advocates for those affected by ovarian cancer, are members of the Registry Steering Committee and were involved in the review, evaluation and selection of the OTP cancer QIs. A consumer representative also sits on this governing committee.

\section{Participants and opt-out process}

Site representatives (clinicians and/or database managers) are responsible for assessing eligibility of their patients for the registry. The registry uses an opt-out method of recruitment whereby participants are informed of their enrolment in the registry unless they exercise their right to withdraw their participation in the registry. Participants can opt-out of the registry at any time either by requesting to be removed from the registry entirely (a 'full' opt-out) or maintaining their medical records but requesting no future contact (a 'partial' opt-out). The adoption of an opt-out method of recruitment is vital for the success of the NGOR. It has been demonstrated in other registries and quality improvement programme that using an opt-in model (ie, obtaining written consent in advance from potential participants) often results in insufficient recruitment of the total eligible population to allow for 


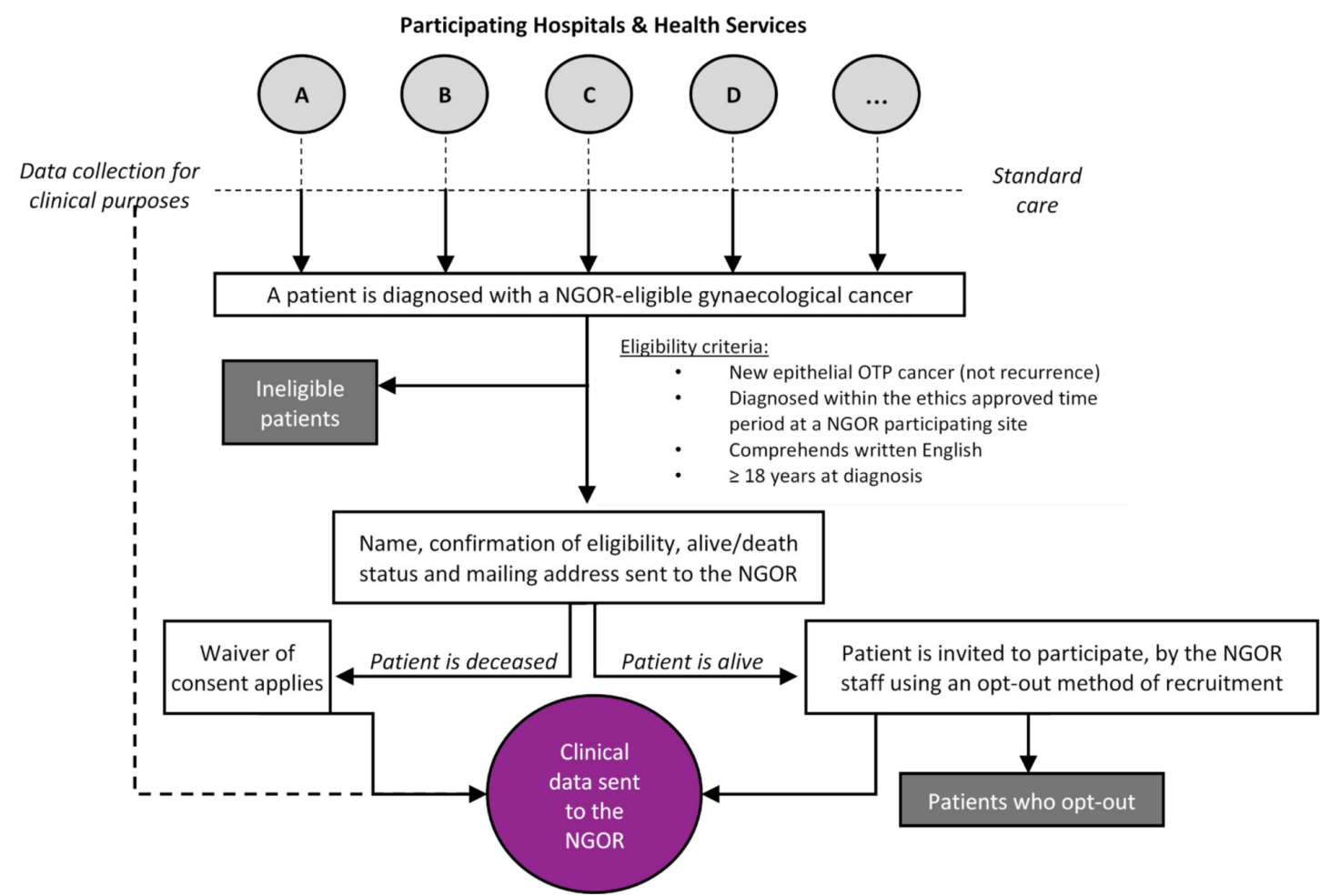

Figure 1 Participant recruitment and data transfer process in the NGOR. NGOR, National Gynae-Oncology Registry; OTP, ovarian, tubal and peritoneal.

meaningful and appropriate assessment of quality of care. Whole population recruitment, or as close to this as possible, also reduces recruitment bias, by ensuring even the sickest and most disadvantaged patients are included. ${ }^{20}$

A waiver of consent for eligible participants who are already deceased by the time the registry is informed of their diagnosis allows for the collection of these participants' medical data. This is important again to ensure whole population capture, and to eliminate bias in the recruitment of the potentially more unwell, that is patients who may have been diagnosed late and/or received care that was not of the same quality as other patients with a similar diagnosis. Thus, exclusion of this subpopulation because of their inability to consent would bias the registry results and limit the ability to fairly riskadjust and benchmark data.

Figure 1 illustrates the NGOR recruitment and data collection pathway.

\section{Quality indicators}

Data collected in the NGOR will be used to inform the QIs developed using current literature, guidelines and specialist expertise. Over time, the NGOR will be in a position to show whether there is any unwarranted variation in care across Australia in women with newly diagnosed ovarian cancer. Only the QIs for the ovarian cancer module have been developed at this stage. The process of QI development commenced in 2015 with a review of current literature and published guidelines, and generated a list of 160 possible QIs. These QIs were expansive and covered a number of domains including diagnosis, staging, pathology, surgery, chemotherapy, radiotherapy and other treatment, management and timeliness of care, palliation and survival. The Steering Committee reviewed this dataset, and developed a shortlist of QIs covering key priority areas. In July 2015, members of ASGO were surveyed about possible QIs for optimal surgical staging and optimal debulking. There were 28 responders out of 54 invited (response rate of 52\%). Results from this survey were considered in subsequent discussions about QIs. During two formal meetings of the Steering Committee and ongoing discussions, a shortlist of nine QIs was created and subsequently revised. These are regularly discussed and modified if appropriate. The current set is listed in table 1 and the process of QI development is illustrated in figure 2 .

\section{Trialling a different method for capturing data}

This is a non-interventional study primarily using previously collected clinical data from pre-existing clinical databases maintained by consulting gynaecologic oncologists and gynaecologic oncology units in major public hospitals across Australia. The registry pilot aimed to demonstrate the feasibility and ease of this predictably more cost-effective and sustainable method of registry operations given the considerable costs associated with manual data collection through medical record review by central, trained data collectors who are required to interpret clinical notes written for a different purpose. This novel method is expected to allow for high-quality, 
Table 1 Quality indicators for the inaugural NGOR module

\section{Number Quality indicator}

$1 \quad$ Percentage of patients with apparent stage I, II or IIIA OTP cancer who are adequately surgically staged.

\section{Data definitions}

'Adequate surgical staging'=where clinically applicable, all of the following procedures were performed:

- Peritoneal washings (and sent for cytology).

- Total abdominal hysterectomy and bilateral salpingooophorectomy (only in cases where fertility preservation is not a priority).

- Infra-colic omentectomy or wedge biopsy.

- Sampling of pelvic lymph nodes.

- Sampling of para-aortic lymph nodes; and.

- Appendicectomy (mucinous tumours only).

$2 \quad$ Percentage of patients with newly diagnosed

OTP cancer who are presented at a multidisciplinary team meeting during which a treatment plan was made.

\section{Percentage of patients who receive a} histological or cytological confirmation of an OTP cancer diagnosis prior to receiving neoadjuvant chemotherapy.
'Neo-adjuvant' chemotherapy here indicates any chemotherapy given to the patient prior to any planned debulking procedure (even when the patient did not subsequently undergo surgery for clinical reasons).

$4 \quad$ Percentage of women with newly diagnosed

OTP cancer who receive first-line

chemotherapy that is platinum-based.

$5 \quad$ Percentage of patients with advanced OTP cancer undergoing primary debulking surgery.

i. have optimal debulking to no macroscopic residual cancer, or

ii. have optimal debulking to $\leq 1 \mathrm{~cm}$ macroscopic residual cancer

6 Percentage of patients with advanced OTP cancer who undergo interval debulking surgery:

i. Have optimal debulking to no macroscopic residual cancer, or

ii. Have optimal debulking to $\leq 1 \mathrm{~cm}$ macroscopic residual cancer.

7 Percentage of major operations for epithelial OTP cancer with an adverse event occurring within 30 days of surgery.

$8 \quad$ Percentage of major operations for epithelial OTP cancer with an adverse event occurring within 30 days of surgery.

9

Percentage of women with newly diagnosed

OTP cancer who receive a referral for, or are offered, genetic testing.
'Advanced'=stages IIB, IIIB, IIIC and IV (International Federation of Gynecology and Obstetrics (FIGO) classification)

'Primary debulking'=debulking surgery performed after 0 cycles of chemotherapy

'Interval debulking'=debulking surgery performed after 3-4 cycles of chemotherapy 


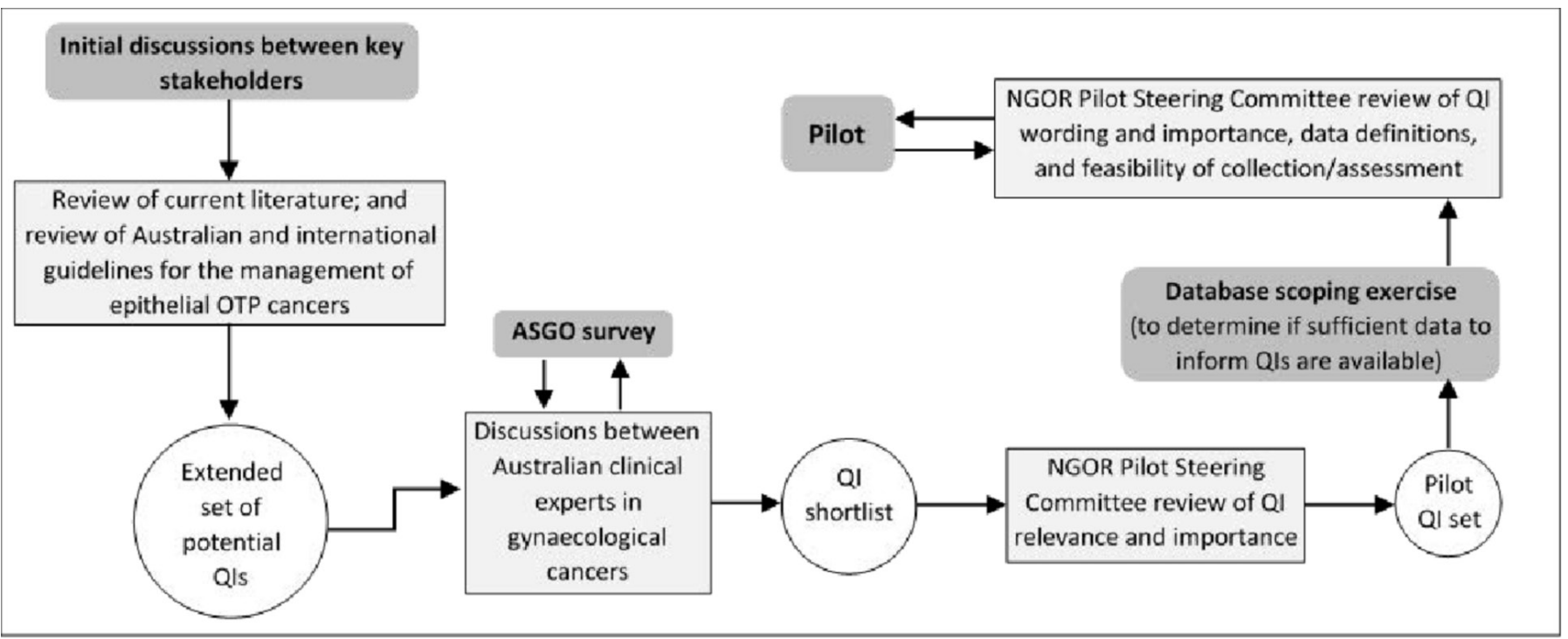

Figure 2 Process of QI development for the inaugural NGOR module. ASGO, Australian Society of Gynaecologic Oncology; NGOR, National Gynae-Oncology Registry; OTP, ovarian, tubal and peritoneal; QI, quality indicator.

consistent data collection in a more time and cost-effective method, avoiding duplication of effort.

A database alignment phase performed in mid-2017 involved the assessment of the various data items required to inform the nine QIs. This found that all the site databases contained sufficient data on the QIs. Following some minor adjustments to these existing datasets, the NGOR will continue to follow this method of data collection.

Data will be collected from the time of diagnosis until the date of death or registry closure, allowing for the comparison of variations in outcome and clinical practice across individual units, across the private versus public sector and regional versus metropolitan centres as well as facilitate national and international benchmarking against agreed best practice.

The NGOR will collect data on women with gynaecological cancers until there is clear and consistent evidence of best practice care provision nationwide. Data collection has already commenced for one module, and remaining modules will commence data collection and participant recruitment in mid- to late-2020

All current participating sites and clinicians involved already collect patient data for clinical purposes and agreed to contribute relevant data items from these databases to the NGOR. Together these account for the majority of public gynaecologic oncology treatment centres in the participating states. Although commencing with the recruitment of these specialist centres may introduce bias to the dataset and not accurately represent the Australian population being treated for these cancers, it has been deemed as the most cost-effective and feasible methodology and will not limit the growth of the registry over time. Other smaller centres without major gynaecologic oncology units, as well as the units in other as-of-yet not participating states will be recruited once registry methodology and each module's QI set has been assessed for usefulness and feasibility of collection. The registry will gradually work towards the ultimate target of total population capture, or as close to this as possible.

Datasets are imported at regular time points into the registry, housed in Monash University's Department of Epidemiology and Preventive Medicine, through a secure portal, and this process is expected to inform most of the registry outputs. In the future, if sites without databases wish to contribute to the registry, the methodology will need to be adapted and may reflect that of other CQRs, or may involve assisting sites to maintain a NGOR standard site-based database which can sync with the registry. Alternatively, sites may choose to enter data directly into the registry. In this instance, limited access to the registry's electronic database will be granted allowing specific site personnel access to only that site's records.

\section{Data cleaning, analysis and reporting}

Data entered into the registry is cleaned by the registry coordinator, and where necessary and appropriate, modified to adhere to registry data structures. Data will be risk-adjusted using additional data items such as comorbidity data, participant age, body mass index and stage at diagnosis, to ensure that fair comparisons between institutions and clinicians can be made. Data will then be aggregated and reported against the predetermined QIs. No identifying or potentially reidentifiable data will be published. Participating sites and clinicians will receive regular benchmarked reports once the CQR has reached maturity, illustrating how their performance in respect to each QI compares with their deidentified counterparts. Outliers (defined as indicator performance that is outside three SDs from the mean performance of their peers) identified in these reports will prompt site-led investigations into causes for variation, and drive improvements in clinical care. This process is illustrated in figure $3{ }^{21}$

An outlier management policy developed by Monash University registry experts will guide the Steering 


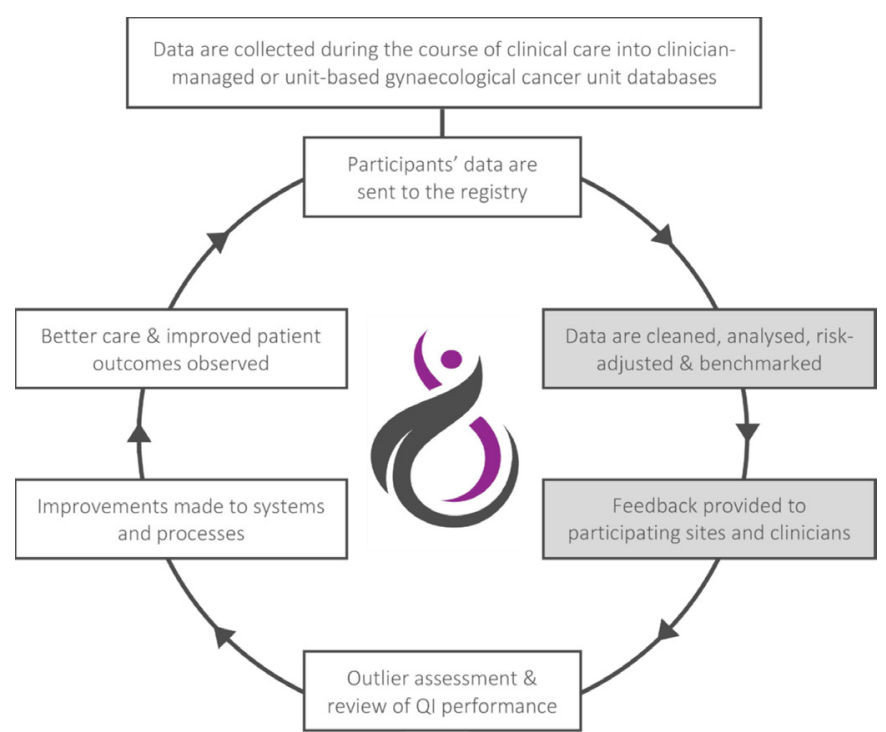

Figure 3 The data process in the NGOR. Adapted from the Australian Commission on Safety and Quality. Boxes shaded in grey represent the actions of the registry, The remaining actions are those of the hospital, health service and/or individual clinician. NGOR, National Gynae-Oncology Registry; QI, quality indicator.

Committee's actions if, a significant and persistently poorly performing outlier is identified, to ensure there is a clear framework for verification, investigation and resolution of the cause. Sites identified as performing poorly against a QI will be provided ample opportunity to review their data, and the registry data and methodology will be audited to ensure that any publicly reported QI performance is a true reflection of care provision and not that of data collection error or misinterpretation of data. Furthermore, the registry Steering Committee will be required to approve requests for data access in the event that researchers, internal or otherwise, wish to access NGOR data for additional research and publication.

\section{ETHICS AND DISSEMINATION}

Data collected for the registry will be routinely reported back to participating sites illustrating their performance against the various QIs, in addition to annual reports that will be publicly available showing registry growth and achievements. It will be the generation and distribution of routine QI reports that the registry will help drive improvements to care provision and to the improvement of outcomes for Australian women with gynaecological cancers.

\section{The future}

The registry is currently in a transition phase, with funding secured for additional modules to be developed and run within the piloted framework, alongside the continuing OTP module. The establishment of the NGOR allows Australia to quantify variation and improve outcomes for patients with gynaecological cancers, and facilitate adherence to best practice care nationally.
Over time, as a greater proportion of the populationof-interest are recruited to the registry, the registry should increase its potential to identify variation across high versus low volume sites, remote versus regional sites and draw other comparisons. In recruiting high-volume sites first, solid evidence of 'best practice' and what is achievable realistically with the greatest resources and specialist involvement, the registry should provide a solid framework against which future lower volume and lessestablished sites can be compared against both in performance against QIs as well as fundamental aspects of clinical management that may be amenable. By providing indisputable evidence of the impact of known barriers to best practice care for remote and low-volume centres, the registry aims to improve care nationwide.

The NGOR Steering Committee intends to incorporate the collection of patient-reported outcomes (PROs) in the future, to compliment the clinical data on quality of care. PROs will provide additional information that can only be obtained from patients about quality of care, and is invaluable to a CQR such as this. Patient-reported outcome measures are not currently used in the registry as the best and more appropriate tool, as well as the method with which they will be incorporated to the registry, and how the data will be used must be determined in a substudy. This is planned for the near future, but could not be performed before the clinical data requirements were finalised.

\section{CONCLUSION}

Until significant advances in diagnostic technology, and the development of more effective therapies for gynaecological cancers become available for routine care, optimising survival for women diagnosed with these diseases will rely on the delivery of timely, evidence-based care. A CQR such as the NGOR is a valuable method for assessing the current quality of care provided to these women, identifying where variation may exist, and prompting service-level change to improve patient outcomes. This is of particular importance in ensuring care is equitable, and working towards uniform clinical quality that does not vary significantly by geographical region or across demographic subgroups.

\section{Author affiliations}

${ }^{1}$ Department of Epidemiology and Preventive Medicine, Monash University, Melbourne, Victoria, Australia

${ }^{2}$ Department of Gynaecological Oncology, Westmead Hospital, Westmead, New South Wales, Australia

${ }^{3}$ University of Sydney, Sydney, NSW, Australia

${ }^{4}$ Division of Obstetrics and Gynaecology, Faculty of Health and Medical Sciences, University of Western Australia, Crawley, Western Australia, Australia

${ }^{5}$ Gynaecological Cancer Research Group, St John of God Subiaco Hospital, Subiaco, Western Australia, Australia

${ }^{6}$ Institute for Health Research, University of Notre Dame Australia, Fremantle, Western Australia, Australia

${ }^{7}$ Ovarian Cancer Australia, Melbourne, Victoria, Australia

${ }^{8}$ Department of Gynaecological Oncology, Mercy Hospital for Women, Heidelberg, Victoria, Australia 
${ }^{9}$ Alfred Health, Melbourne, VIC, Australia

${ }^{10}$ Gynaecological Oncology, Obstetrics and Gynaecology Clinical Institute, Epworth, Melbourne, Victoria, Australia

Acknowledgements The authors would like to acknowledge the National GynaeOncology Registry Steering Committee, Ms Annie Stenlake from Westmead Hospital, and Dr Ri Scarborough and Ms Amy Hatton from the Cancer Research Programme at Monash University.

Contributors $\mathrm{NH}$ is the coordinator of the National Gynae-Oncology Registry (NGOR) and has prepared the protocol for this project along with input from the authored clinicians. NH prepared the draft of this paper and received feedback and wording changes from all the listed authors (AB PC SHe SHy YL JRZ and RR). $\mathrm{RR}$ is the clinical lead in this project and has been instrumental in supporting and initiating the NGOR, particularly in regards to funding. JRZ is the academic lead in this project and head of the Cancer Research Program at Monash University, JRZ has been involved with this project since its inception and secured funding for the coordinator role and other NGOR supporting roles.

Funding Funding for this project is courtesy of the Australian Society of Gynaecologic Oncologists (ASGO), the CASS Foundation (CASS) and Ovarian Cancer Australia (OCA). The funders have not had any input in the collection, analysis or interpretation of data, the writing of this paper or the decision for publication.

Competing interests None declared.

Patient consent for publication Not required.

Ethics approval The National Gynae-Oncology Registry (NGOR has been approved by the Monash Health Human Research Ethics Committee (HREC) under the National Mutual Acceptance Scheme (NMA HREC Reference Number: HREC/17/ MonH/198), as well as by the Monash University HREC, the University of Tasmania HREC, the Mercy Health HREC and the Epworth HealthCare HREC. All participating sites have granted site governance approval.

Provenance and peer review Not commissioned; externally peer reviewed.

Open access This is an open access article distributed in accordance with the Creative Commons Attribution Non Commercial (CC BY-NC 4.0) license, which permits others to distribute, remix, adapt, build upon this work non-commercially, and license their derivative works on different terms, provided the original work is properly cited, appropriate credit is given, any changes made indicated, and the use is non-commercial. See: http://creativecommons.org/licenses/by-nc/4.0/.

ORCID iD

Natalie Heriot http://orcid.org/0000-0002-4189-9433

\section{REFERENCES}

1 Marsden DE, Friedlander M, Hacker NF. Current management of epithelial ovarian carcinoma: a review. Semin Surg Oncol 2000;19:11-19.

2 Australian Institute of Health and Welfare. Gynaecological cancers in Australia: an overview. Canberra: AlHW, 2012.
3 Jordan SJ, Francis JE, Nelson AE, et al. Pathways to the diagnosis of epithelial ovarian cancer in Australia. Med J Aust 2010;193:326-30.

4 Anuradha S, Webb PM, Blomfield P, et al. Survival of Australian women with invasive epithelial ovarian cancer: a population-based study. Med J Aust 2014;201:283-8.

5 Grossi M, Quinn MA, Thursfield VJ, et al. Ovarian cancer: patterns of care in Victoria during 1993-1995. Med J Aust 2002;177:11-16.

6 Australian Commission on Safety and Quality in Health Care. Framework for Australian clinical quality registries. Sydney: ACSQHC, 2014.

7 Australian Institute of Health and Welfare. Cancer in Australia 2019. Canberra: AlHW, 2019.

8 Lheureux S, Braunstein M, Oza AM. Epithelial ovarian cancer: evolution of management in the era of precision medicine. $C A$ Cancer J Clin 2019:69:280-304.

9 Holschneider $\mathrm{CH}$, Berek JS. Ovarian cancer: epidemiology, biology, and prognostic factors. Semin Surg Oncol 2000;19:3-10.

10 Maringe C, Walters S, Butler J, et al. Stage at diagnosis and ovarian cancer survival: evidence from the International cancer benchmarking partnership. Gynecol Oncol 2012;127:75-82.

11 World Health Organisation. World cancer report 2014. Lyon: WHO, 2014.

12 Soong TR, Howitt BE, Horowitz N, et al. The fallopian tube, "precursor escape" and narrowing the knowledge gap to the origins of high-grade serous carcinoma. Gynecol Oncol 2019;152:426-33.

$13 \mathrm{Kim} \mathrm{SI}$, Lee M, Kim HS, et al. Effect of BRCA mutational status on survival outcome in advanced-stage high-grade serous ovarian cancer. J Ovarian Res 2019;12:40.

14 Aletti GD, Peiretti M. Quality control in ovarian cancer surgery. Best Pract Res Clin Obstet Gynaecol 2017;41:96-107.

15 Bristow RE, Palis BE, Chi DS, et al. The National cancer database report on advanced-stage epithelial ovarian cancer: impact of hospital surgical case volume on overall survival and surgical treatment paradigm. Gynecol Oncol 2010;118:262-7.

16 du Bois A, Reuss A, Pujade-Lauraine E, et al. Role of surgical outcome as prognostic factor in advanced epithelial ovarian cancer: a combined exploratory analysis of 3 prospectively randomized phase 3 multicenter trials: by the Arbeitsgemeinschaft Gynaekologische Onkologie Studiengruppe Ovarialkarzinom (AGOOVAR) and the Groupe d'Investigateurs Nationaux pour les Etudes des cancers de l'Ovaire (GINECO). Cancer 2009;115:1234-44.

17 Bristow RE, Tomacruz RS, Armstrong DK, et al. Survival effect of maximal cytoreductive surgery for advanced ovarian carcinoma during the platinum era: a meta-analysis. $J$ Clin Oncol 2002:20:1248-59.

18 Vernooij F, Heintz P, Witteveen E, et al. The outcomes of ovarian cancer treatment are better when provided by gynecologic oncologists and in specialized hospitals: a systematic review. Gynecol Oncol 2007;105:801-12.

19 Bonte A-S, Luyckx A, Wyckmans L, et al. Quality indicators for the management of endometrial, cervical and ovarian cancer. Eur J Surg Oncol 2019;45:528-37.

20 Evans SM, Loff B, Cameron PA. Clinical registries: the urgent need to address ethical hurdles. Med J Aust 2013;198:134-5.

21 Australian Commission on Safety and Quality in Health Care. National arrangements for clinical quality registries. Available: https:// www.safetyandquality.gov.au/our-work/national-arrangementsclinical-quality-registries 